\title{
Efectividad de las intervenciones familiares en centros geriátricos. Una revisión sistemática
}

\author{
Effectiveness of family interventions in nursing homes. \\ A systematic review
}

\author{
A. Villanueva Lumbreras', C. García-Vivar ${ }^{2}$, N. Canga Armayor ${ }^{2}$, A. Canga Armayor ${ }^{2}$
}

\section{RESUMEN}

La institucionalización en un centro geriátrico supone en muchas ocasiones un acontecimiento estresante, tanto para la persona mayor dependiente como para su familia. Durante el ingreso, especialmente en los primeros meses, la familia puede tener sentimientos de pérdida, tristeza, fracaso o culpabilidad. En este contexto, resulta fundamental el apoyo y orientación de los profesionales del centro, a través de intervenciones efectivas que aborden las necesidades tanto del residente como de su familia.

El objetivo de esta revisión sistemática es identificar cuáles son las intervenciones familiares más efectivas para ayudar a las familias durante el proceso de institucionalización en un centro gerontológico.

Se han identificado dos tipos de intervenciones: aquellas que se centran en la relación familia-personal sanitario y, las que se enfocan a grupos de apoyo a familiares, siendo estas últimas las más efectivas. Por otro lado, la mayoría de intervenciones tienen un enfoque individual, centrándose en el cuidador principal, sin abordar la unidad familiar. Por último, hay una escasez de artículos de calidad que presenten resultados sobre intervenciones familiares en el ámbito geriátrico, identificándose una ausencia de estos estudios en el contexto español.

Palabras clave. Cuidador familiar. Centro geriátrico. Intervenciones.

\begin{abstract}
The process of nursing home placement can be a stressful event for both the dependent elderly person and the family. During admission, especially the first few months, the family may suffer feelings of loss, sadness, failure and guilt. In this context, support and guidance from health professionals are essential to address the needs of residents and families through effective interventions.

The aim of this systematic review is to identify the most effective interventions to help families during the process of institutionalization of a relative in a nursing home.

Two types of family interventions were identified: those that focused on family-staff relationship and those that focused on family support groups, the latter being the most effective. On the other hand, most interventions have an individualistic approach, focusing on the primary caregiver. Finally, there is a shortage of quality studies that present the results of family interventions in the geriatric field and also a lack of such studies in the Spanish context.
\end{abstract}

Keywords. Family caregiver. Nursing home. Interventions.
1. Centro Psicogeriátrico Landazábal. Burlada.

2. Facultad de Enfermería. Universidad de Navarra. Pamplona.

Recepción: 8 de septiembre de 2014

Aceptación provisional: 3 de noviembre de 2014

Aceptación definitiva: 19 de enero de 2015

\section{Correspondencia:}

Amaya Villanueva Lumbreras

Centro Psicogeriátrico Landazábal

C/ Landazábal 2

31600 Burlada. Navarra

E-mail:amayavl@hotmail.com 


\section{INTRODUCCIÓN}

Los avances sanitarios y sociales de los países occidentales han originado un incremento del número de personas mayores de 65 años, dando lugar a uno de los grandes logros de estos países, el envejecimiento de la población ${ }^{1}$. Esta realidad origina paralelamente un aumento en el número de personas dependientes, puesto que las limitaciones de la capacidad funcional se amplían en los grupos de edad superiores ${ }^{2}$. La consecuencia es que estas personas requieren de los llamados "Cuidados de larga duración” (Long-TermCare).

En España, al igual que en otros países del sur de Europa, el cuidado de las personas dependientes es asumido principalmente por la familia ${ }^{2-3}$. Sin embargo, los acontecimientos sociales actuales, marcados por la progresiva incorporación de la mujer al mercado laboral, junto con los cambios en la estructura familiar, hacen que cada vez sea más complicado mantener esta tendencia ${ }^{2-5}$.

$\mathrm{Al}$ mismo tiempo los cuidados de larga duración de un familiar dependiente tienen un gran impacto en la unidad familiar, aumentando progresivamente sus necesidades de cuidado físico, emocional y espiritual $^{3-5}$.

Ante esta perspectiva, en los últimos años la oferta de servicios sociales dirigidos a personas mayores ha experimentado un considerable incremento. Datos actuales reflejan cómo un $73,34 \%$ de las plazas ofertadas en residencias geriátricas en España son para personas en situación de dependencia ${ }^{6}$.

En este sentido conviene señalar que la institucionalización en un centro geriátrico puede ser uno de los acontecimientos más importantes en la vida de una persona anciana y de su familia ${ }^{7}$. Es un proceso que puede ocasionar sentimientos ambivalentes. Generalmente produce alivio en los familiares cuidadores, ya que no tienen que procurar los cuidados básicos a su familiar y, pueden dedicar ese tiempo al establecimiento de una relación de calidad con su ser querido ${ }^{8-9}$. No obstante, en la mayoría de las ocasiones la institucionalización no disminuye la carga y el estrés de los cuidadores informales sino que los exacerba, evidenciándose sentimientos de pérdida, tristeza, fracaso o culpabilidad ${ }^{9}$. Siendo así que la familia sigue implicándose en el cuidado de su familiar dependiente, queriendo participar activamente en el centro ${ }^{10-11}$. Por ello, se considera importante que los profesionales que trabajan en los centros geriátricos (enfermeras, geriatras, trabajadores sociales, psicólogos y auxiliares de enfermería) conozcan las necesidades de la familia y los retos a los que ésta se enfrenta durante la institucionalización. $\mathrm{El}$ fin es apoyar a la familia en esta etapa de transición, siendo necesaria una atención basada en intervenciones efectivas enfocadas a ayudar a la unidad familiar, cuando un miembro dependiente es institucionalizado, y llevadas a cabo por profesionales formados en el cuidado a la unidad familiar. Sin embargo, cabe señalar la escasez de intervenciones desarrolladas en centros geriátricos, con un enfoque centrado en la familia. Este escenario resulta llamativo ya que los estudios evidencian que la implicación de la familia en dichos centros tiene efectos beneficiosos, tanto para los residentes como para la propia familia ${ }^{8-11}$.

Por todo lo expuesto se plantea este estudio cuyo objetivo es conocer y evaluar la efectividad de las intervenciones familiares desarrolladas en centros gerontológicos.

\section{MATERIAL Y MÉTODOS}

Se realizó una revisión sistemática de estudios publicados en las bases de datos Pubmed, CINAHL, PsycInfo, y Cochrane Library, para poder responder al objetivo propuesto. Se estableció una búsqueda siguiendo la estrategia PICOS: participantes, intervenciones, comparación, resultados y diseño del estudio ${ }^{12}$. La búsqueda se llevó a cabo entre junio y agosto de 2014. Se estableció únicamente como límite el idioma, seleccionando aquellos artículos que estuviesen redactados en inglés, francés y castellano.

Se incluyeron los siguientes términos Mesh: family caregiver, intervention y nursing home. Se combinaron entre ellos y con 


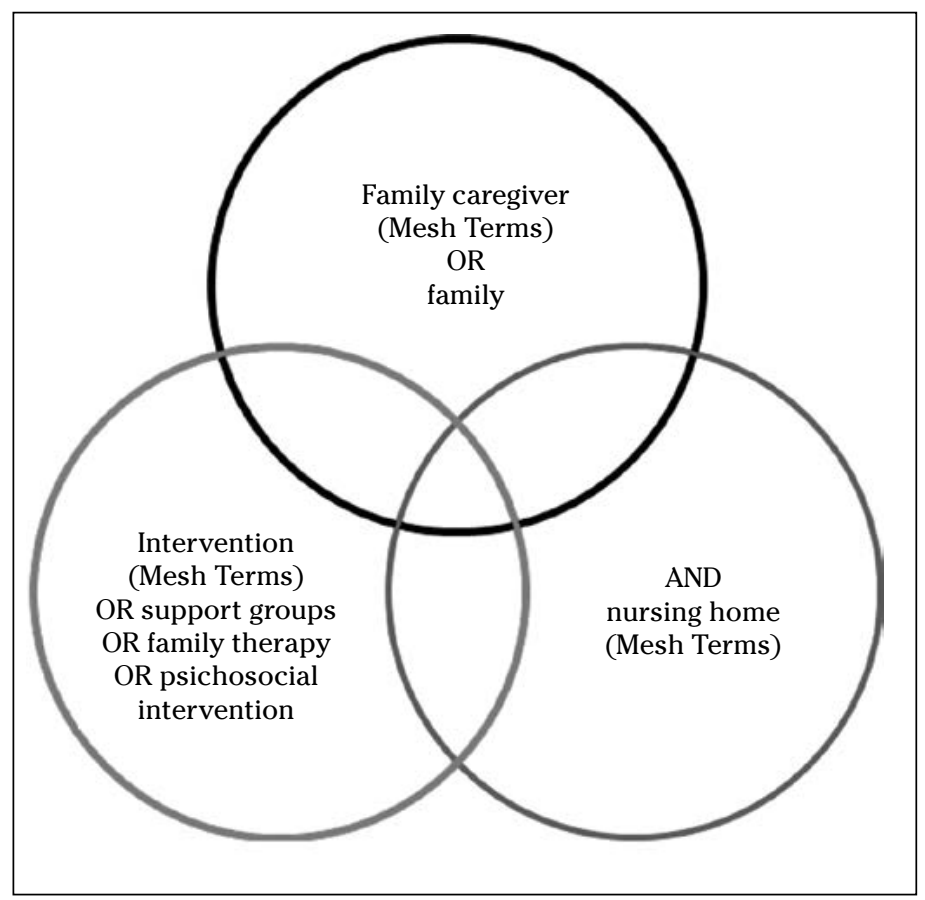

Figura 1. Diagrama de la estrategia de búsqueda

otros sinónimos empleando los operadores booleanos "AND" y "OR" para incluir posibles variaciones utilizadas en la literatura, como se muestra en la figura 1.

Los criterios de selección de los artículos fueron:

Criterios de inclusión: estudios experimentales y cuasi-experimentales sobre intervenciones dirigidas a familias con un familiar institucionalizado en un centro geriátrico.

Criterios de exclusión: estudios descriptivos o estudios cualitativos. Estudios que no incluyesen entre sus participantes a la familia de residentes en centros geriátricos.

La selección de los estudios fue llevada a cabo por un único investigador. A continuación dos revisores independientes analizaron la relevancia de los estudios seleccionados.

Para analizar la calidad de los estudios, se utilizó la herramienta Cochrane ${ }^{13}$ que valora seis ítems: formación de los grupos, asignación aleatoria, enmascaramiento, índice de abandono, datos incompletos de los resultados y otros sesgos. Conforme se iba revisando y analizando cada apartado, se determinaba si existía riesgo de sesgo en los estudios y se especificaba si éste era bajo, elevado o no había suficiente información para determinarlo. A continuación se clasificaban según el nivel de evidencia. Nivel A si existía aleatorización con grupo control y enmascaramiento; Nivel B si existía aleatorización sin enmascaramiento claro y definido; y Nivel C si no se había llevado a cabo la aleatorización o se había realizado sin un adecuado enmascaramiento ${ }^{14}$.

\section{RESULTADOS}

\section{Selección de los artículos}

Se identificaron 326 artículos, a los que se añadieron cuatro más, mediante la técnica de "snowballing", sumando un total 
de 330. Se eliminaron los duplicados y, tras una primera lectura de los títulos y resúmenes, se seleccionaron 64 artículos. Éstos se analizaron en profundidad eligiéndose 18 , de los cuales se eliminaron 11 por no cumplir con los criterios de inclusión (Ta- bla 1). Finalmente fueron siete artículos los incluidos en la revisión sistemática, para analizar la efectividad de las intervenciones familiares desarrolladas en centros geriátricos (Fig. 2).

Tabla 1. Lista artículos excluidos por no cumplir los criterios de inclusión

\begin{tabular}{|c|}
\hline Artículo \\
\hline $\begin{array}{l}\text { Sury L, Burns K, Brodaty H. Moving in: Adjustment of peo- } \\
\text { ple living with dementia going into a nursing home and their } \\
\text { families. Int Psychogeriatr 2013; 25: 867-876. }\end{array}$ \\
\hline $\begin{array}{l}\text { Gaugler J, Anderson K, Holmes H. Family-Based Intervention } \\
\text { in Residential Long-Term Care. Marriage Fam Rev 2005; 37: } \\
\text { 45-62. }\end{array}$ \\
\hline $\begin{array}{l}\text { Nazir A, Unroe K, Tegeler M, Khan B, Azar J, Boustani M. Sys- } \\
\text { tematic review of interdisciplinary interventions in nursing } \\
\text { homes. J Am Med Dir Assoc } 2013 \text { Jul;14: 471-478. }\end{array}$ \\
\hline $\begin{array}{l}\text { Gaugler J, Roth D, Haley W, Mittelman M. Can counseling and } \\
\text { support reduce burden and depressive symptoms in care- } \\
\text { givers of people with Alzheimer's disease during the transi- } \\
\text { tion to institutionalization? Results from the New YorkUni- } \\
\text { versity caregiver intervention study. J Am Geriatr Soc } 2008 \text {; } \\
56: 421-428 \text {. }\end{array}$ \\
\hline $\begin{array}{l}\text { Drysdale A, Nelson C, Wineman N. Families need help too: } \\
\text { group treatment for families of nursing home residents. Clin } \\
\text { Nurse Spec 1993; 7: 130-134. }\end{array}$ \\
\hline $\begin{array}{l}\text { Jablonski R, Reed D, Maas M. "Care intervention for older } \\
\text { adults with Alzheimer's disease and related dementias: ef- } \\
\text { fect of family involvement on cognitive and functional out- } \\
\text { comes in nursing homes", J Gerontol Nurs } 2005 ; 31: 38-48 \text {. }\end{array}$ \\
\hline
\end{tabular}

Tsai H, Tsai. Changes in depressive symptoms, social support, and loneliness over 1 year after a minimum 3-month videoconference program for older nursing home residents. J Med Internet Res 2011; 13:e93.

Dijkstra A. Family participation in care plan meetings: promoting a collaborative organizational culture in nursing homes. J Gerontol Nurs 2005; 33: 22-31

Peak T. Families and the nursing home environment: adaptation in a group context. J GerontolSoc Work 2000; 1: 51-66.

Van der Ploeg E, Camp C, Eppingstall B, Runci S, O'Connor D. The study protocol of a cluster-randomised controlled trial of family-mediated personalised activities for nursing home residents with dementia 2012. BMC Geriatr; 12: 2.

Crispi E, Heitner G. An activity-based intervention for caregivers and residents with dementia in nursing homes 2002. Act Adapt Aging; 26: 61-72.

\section{Motivo de exclusión}

Revisión sistemática que analiza los factores que facilitan la adaptación del residente y su familia a la residencia geriátrica. No hay intervención.

Revisión narrativa sobre intervenciones familiares en centros geriátricos.

Revisión sistemática acerca de intervenciones interdisciplinares en centros geriátricos. Los participantes son los profesionales.

Se descarta ya que se escoge un artículo del mismo estudio que analiza los resultados de la intervención.

No analizan estadísticamente los resultados de la intervención. Estudio descriptivo.

Descartado porque forma parte del estudio de Mass (2004) el cual ya se ha incluido y porque se centra en evaluar los resultados de la intervención únicamente en el estado cognitivo y funcional de los residentes con demencia.

Se ha seleccionado para el análisis la intervención, detallada en su artículo publicado en 2010 . En este artículo se profundiza en otros aspectos de la intervención no relevantes para el estudio.

No analizan estadísticamente los resultados de la intervención. Estudio descriptivo.

No analizan estadísticamente los resultados de la intervención. Estudio descriptivo.

Estudio descriptivo.

Estudio descriptivo. 
Palabras clave: nursing home, family, intervention

Límites: idiomas inglés, castellano y francés

Bases: Pubmed 84, Cinhal 55, Psylnfo 88, Cochrane Library 99
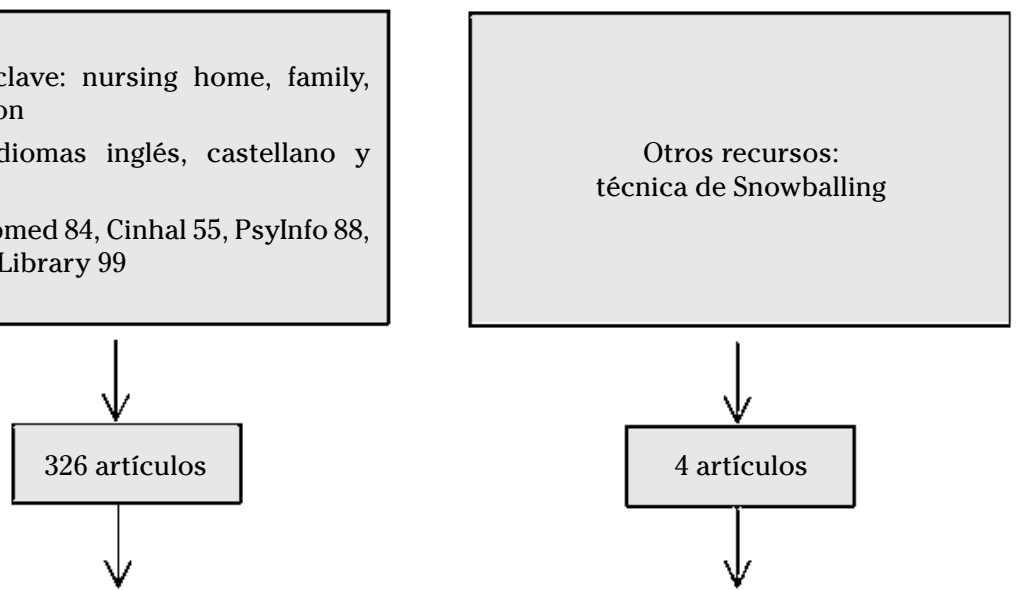

4 artículos

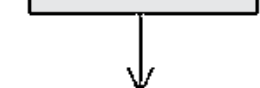

$\sqrt{ }$

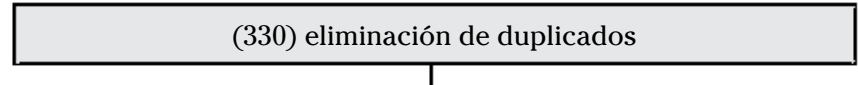

$\sqrt{ }$

\section{6 artículos}
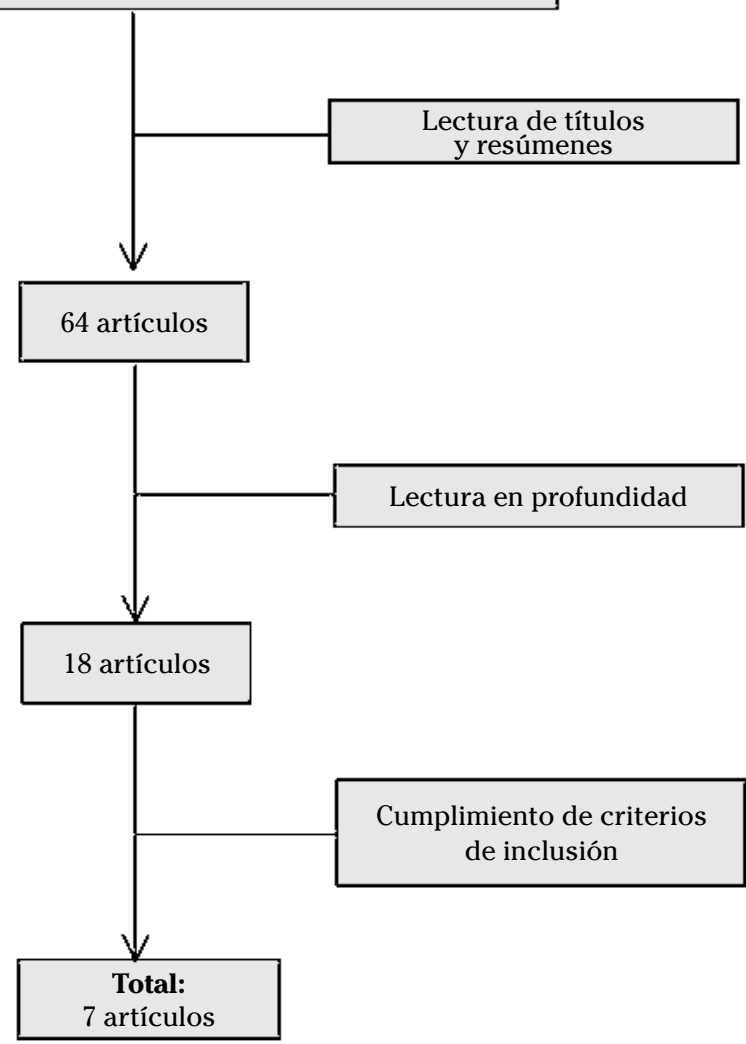

Figura 2. Proceso de selección de estudios. 


\section{Análisis de los estudios}

De los siete artículos seleccionados, cinco son estudios experimentales ${ }^{15,17-20}$ y dos son cuasi-experimentales ${ }^{16,21}$. Los participantes de seis de los estudios son cuidadores principales de personas institucionalizadas ${ }^{15-20}$. Además, en tres de los seis artículos, incluyen también a los profesionales del centro (enfermeras y auxiliares de enfermería $)^{15-16,18}$. Por último, en el estudio de Tsai y col $^{21}$ los sujetos participantes son los residentes del centro.

Todos las investigaciones, incluidas en la revisión, evalúan intervenciones familiares de tipo psicosocial (Tabla 2). Éstas se dividen en dos grupos: aquellas que trabajan la relación entre los profesionales de los centros y las familias de los residentes y las enfocadas a los cuidadores principales de los residentes.

Los estudios que se enfocan en la relación entre los profesionales y los familiares están basados en intervenciones que llevan acabo programas formativos sobre técnicas y estrategias de comunicación, de resolución de conflictos o de afrontamiento que faciliten la relación entre ambos grupos.

De este modo, Pillemer y col $^{15}$, a través de un estudio controlado aleatorizado evaluaron la efectividad de una intervención educativa centrada en mejorar la cooperación y la comunicación entre los familiares y el personal de centros geriátricos. Para ello realizaron unas sesiones grupales en las que trabajaban técnicas de comunicación y habilidades de resolución de conflictos. Utilizaron diversas metodologías como el rol-playing o el brainstorming.

Para analizar la efectividad de la intervención, evaluaron las variables: nivel de empatía, percepción de conflictos, sentimientos de depresión y carga en las familias, o la intención de dejar el trabajo por parte de los profesionales. Tras el análisis de los datos se objetivó un aumento de la empatía de los familiares hacia los profesionales en el grupo que recibió la intervención. Además disminuyeron los conflictos entre los profesionales y los familiares que la recibieron.
En relación a los posibles sesgos del estudio, se observa que la asignación a los grupos de intervención y control fue aleatoria. En el artículo no se explica si el enmascaramiento fue ciego, doble ciego o triple, siendo este aspecto, sin embargo, clave para conocer el riesgo de sesgo de aleatorización que pudiese presentar.

Uno de sus principales problemas, que además es común al resto de las intervenciones revisadas, es que la intervención presentó un alto índice de abandono por parte de los participantes. Sin embargo, los investigadores manejaron correctamente esta pérdida llevando a cabo una sesión reducida con aquellos participantes que no pudieron terminar el programa. De este modo se puede concluir que incluyeron a todos los participantes identificados al inicio, lo cual evitaría sesgos. Por tanto, la aleatorización al grupo control e intervención, junto con la correcta manipulación de los participantes perdidos, asegura un riesgo bajo de sesgo. Esto incrementa el grado de evidencia del estudio, aunque el desconocimiento acerca del tipo de enmascaramiento que se llevó a cabo implica que se le otorgue un grado $B$ de evidencia.

Robinson y coll $^{17}$ evaluaron a través de un estudio aleatorio controlado, la intervención desarrollada anteriormente por Pillemer y $\mathrm{col}^{15}$, pero con la diferencia de que los residentes de los familiares, que participaron en el estudio, presentaban exclusivamente demencia. El objetivo del estudio de Robinson y coll $^{17}$ era mejorar la comunicación y la cooperación entre ambos grupos mediante la implementación de una intervención psicosocial. Los resultados obtenidos fueron similares al estudio de Pillemer. En los profesionales no se obtuvieron diferencias significativas entre el grupo que recibió la intervención y el grupo control. En cambio, los familiares que la recibieron mejoraron su capacidad de comunicarse con los profesionales.

En este estudio existe aleatorización de los participantes pero, de nuevo, no se especifica si el enmascaramiento fue doble o triple. Tuvieron un elevado índice de abandono entre los familiares (51\%) que manejaron de forma correcta para evitar 
Tabla 2. Características de los artículos seleccionados.

\begin{tabular}{|c|c|c|c|c|c|c|}
\hline $\begin{array}{l}\text { Autor } \\
\text { (año) }\end{array}$ & Participantes & Objetivo & Diseño & Resultados & $\begin{array}{c}\text { Riesgo } \\
\text { de sesgo }\end{array}$ & $\begin{array}{c}\text { Nivel de } \\
\text { evidencia }\end{array}$ \\
\hline $\begin{array}{l}\text { Pillmer y } \\
\text { col }^{15} \\
(2003)\end{array}$ & $\begin{array}{l}932 \text { familiares } \\
655 \\
\text { profesionales }\end{array}$ & $\begin{array}{l}\text { Disminuir los conflictos } \\
\text { y los síntomas de } \\
\text { depresión, mejorar la } \\
\text { empatía y la cooperación } \\
\text { entre ambos grupos. }\end{array}$ & $\begin{array}{l}\text { Controlado } \\
\text { aleatorizado }\end{array}$ & $\begin{array}{l}\text { En familiares: } \\
\text { aumentó la } \\
\text { empatía con } \\
\text { el personal y } \\
\text { disminuyó su } \\
\text { percepción de } \\
\text { conflictos. } \\
\text { Intervención poco } \\
\text { efectiva en el } \\
\text { personal }\end{array}$ & Bajo & B \\
\hline $\begin{array}{l}\text { Mass y } \\
\mathrm{col}^{16} \\
(2004)\end{array}$ & $\begin{array}{l}185 \text { familiares } \\
845 \\
\text { profesionales }\end{array}$ & $\begin{array}{l}\text { Involucrar a los familiares } \\
\text { en el centro, disminuir } \\
\text { sus sentimientos de } \\
\text { culpabilidad y pérdida. } \\
\text { Mejorar las reacciones } \\
\text { emocionales del personal } \\
\text { hacia el residente y } \\
\text { familiares. }\end{array}$ & $\begin{array}{l}\text { Cuasi- } \\
\text { experimental }\end{array}$ & $\begin{array}{l}\text { Mayor } \\
\text { involucración de } \\
\text { familiares en el } \\
\text { centro. Menor } \\
\text { culpabilidad. } \\
\text { Mejor reacción del } \\
\text { personal hacia los } \\
\text { familiares }\end{array}$ & $\begin{array}{l}\text { No } \\
\text { aplicable }\end{array}$ & $\begin{array}{l}\text { No } \\
\text { aplicable }\end{array}$ \\
\hline $\begin{array}{l}\text { Ducharme } \\
\text { y col }^{18} \\
(2005)\end{array}$ & 137 familiares & $\begin{array}{l}\text { Disminuir el estrés y } \\
\text { la carga del cuidador. } \\
\text { Mejorar su capacidad } \\
\text { de afrontamiento y } \\
\text { comunicación con el } \\
\text { personal. }\end{array}$ & $\begin{array}{l}\text { Controlado } \\
\text { aleatorizado }\end{array}$ & No significativos & Bajo & B \\
\hline $\begin{array}{l}\text { Robinson y } \\
\text { col }^{17} \\
(2007)\end{array}$ & $\begin{array}{l}491 \text { familiares } \\
412 \\
\text { profesionales }\end{array}$ & $\begin{array}{l}\text { Involucrar al familiar } \\
\text { en el centro y facilitar } \\
\text { su comunicación con el } \\
\text { personal. }\end{array}$ & $\begin{array}{l}\text { Controlado } \\
\text { aleatorizado }\end{array}$ & $\begin{array}{l}\text { Efectivo para } \\
\text { facilitar la } \\
\text { comunicación de } \\
\text { los familiares con } \\
\text { el personal. }\end{array}$ & Bajo & B \\
\hline $\begin{array}{l}\text { Gaugler y } \\
\text { col }^{19} \\
(2011)\end{array}$ & 210 familiares & $\begin{array}{l}\text { Disminuir los síntomas } \\
\text { depresivos y la carga del } \\
\text { cuidador tras el ingreso } \\
\text { en un centro }\end{array}$ & $\begin{array}{l}\text { Controlado } \\
\text { aleatorizado }\end{array}$ & $\begin{array}{l}\text { Reducción } \\
\text { de síntomas } \\
\text { depresivos y carga } \\
\text { del cuidador. }\end{array}$ & Bajo & B \\
\hline $\begin{array}{l}\text { Davies y } \\
\text { col }^{20} \\
(2011)\end{array}$ & 46 familiares & $\begin{array}{l}\text { Disminuir los } \\
\text { sentimientos de } \\
\text { culpabilidad, depresión, } \\
\text { carga del cuidador } \\
\text { y conflictos con el } \\
\text { personal. También } \\
\text { aumentar la satisfacción } \\
\text { del personal con el } \\
\text { centro. }\end{array}$ & $\begin{array}{l}\text { Controlado } \\
\text { aleatorizado }\end{array}$ & $\begin{array}{l}\text { Disminuyó los } \\
\text { sentimientos de } \\
\text { culpabilidad de } \\
\text { los familiares y los } \\
\text { conflictos con el } \\
\text { personal. }\end{array}$ & Alto & B \\
\hline $\begin{array}{l}\text { Tsai y col }{ }^{21} \\
(2011)\end{array}$ & 57 residentes & $\begin{array}{l}\text { Disminuir los síntomas } \\
\text { de depresión y soledad } \\
\text { del residente y aumentar } \\
\text { la involucración de sus } \\
\text { familiares con éste. }\end{array}$ & $\begin{array}{l}\text { Cuasi- } \\
\text { experimental }\end{array}$ & $\begin{array}{l}\text { Disminuyó } \\
\text { los síntomas } \\
\text { de soledad } \\
\text { y depresión. } \\
\text { Aumentó el apoyo } \\
\text { social. }\end{array}$ & $\begin{array}{l}\text { No } \\
\text { aplicable }\end{array}$ & $\begin{array}{l}\text { No } \\
\text { aplicable }\end{array}$ \\
\hline
\end{tabular}


posibles sesgos. Por tanto consideramos que, al igual que el estudio predecesor tiene un riesgo bajo de sesgo y un grado B de recomendación.

Un tercer trabajo, que incluye como participantes a familiares y profesionales, es el desarrollado por Mass y col $^{16}$. Este estudio cuasi-experimental evaluó una intervención psicosocial centrada en mejorar la relación entre los profesionales y los familiares. Para ello llevaron a cabo un programa educativo a través del cual les enseñaron técnicas de negociación y colaboración. En este caso, la intervención resultó ser efectiva para modificar la percepción negativa de los profesionales acerca de su relación con los familiares de los residentes. Asimismo, redujo los sentimientos de pérdida y sufrimiento de los familiares.

Conviene señalar que no se pudo analizar el riesgo de sesgo con la herramienta Cochrane, ya que el diseño del estudio era cuasi-experimental. Sin embargo, se ha determinado que la calidad del estudio es adecuada al presentar rigor en la metodología utilizada. Aunque no se debe obviar que el tipo de diseño utilizado impide su replicación en otros contextos.

A continuación, pasamos a analizar el otro grupo de estudios. Aquellos que están basados en intervenciones familiares que se dirigen a los cuidadores principales de los residentes.

Ducharme y $\mathrm{col}^{18}$ llevaron a cabo un estudio controlado aleatorizado para evaluar la eficacia de una intervención enfocada en los hijos de ancianos con demencia institucionalizados. La intervención consistió en un programa educacional que enseñaba a los familiares a expresar sus sentimientos, a afrontar la nueva situación de la institucionalización de su familiar y, a reorganizar su vida tras el ingreso de éste. En este caso no se encontraron diferencias significativas entre los participantes que recibieron la intervención y los que no la recibieron. En el estudio, aunque existe aleatorización, no queda especificado si hubo enmascaramiento doble ciego o triple. Esto puede dar lugar a dudas e incurrir en un posible sesgo de aleatorización. También nos encontramos con un alto índice de abandono.
Para evitar que este hecho alterase los resultados compararon los participantes que abandonaron con los que continuaron en el estudio y vieron que no había diferencias significativas. Una de las principales causas de abandono por parte de los participantes fue la falta de tiempo para acudir a las sesiones. Por todo ello, consideramos que estamos ante un estudio con un riesgo bajo, y se considera que presenta un grado $B$ de evidencia.

En su estudio Gaugler y col ${ }^{19}$ evaluaron, a través de un diseño aleatorio controlado, si una intervención que proporcionaba apoyo y consejo reducía los síntomas de depresión y carga de los familiares de ancianos con demencia, durante el proceso de transición a la institucionalización. Para ello los investigadores llevaron a cabo varias sesiones en las que se impartía asesoramiento familiar e individual, completado con grupos de apoyo. El análisis de los datos evidenció que la intervención reducía los síntomas de depresión y carga en aquellos participantes que recibieron la intervención.

La asignación al grupo control e intervención fue aleatoria pero no se especifica si el enmascaramiento fue ciego, doble o triple. Podría haber riesgo de sesgo en los resultados ya que no puntualizan si tuvieron en cuenta a veintiún participantes que abandonaron el estudio. También puede aumentar el riesgo de sesgo el hecho de que la muestra podría no ser representativa de la población, puesto que no tiene diversidad de raza o etnia, característica del país en el que se desarrolla la intervención, Estados Unidos. Aun así, el diseño del estudio y la muestra son adecuados. Por tanto se considera que existe un riesgo de sesgo bajo y presenta un grado de evidencia $B$.

En un tercer trabajo, Davies y $\mathrm{col}^{20}$ evaluaron a través de un estudio aleatorizado controlado la efectividad de una intervención psicosocial que facilitaba la adaptación de los familiares tras el ingreso de su familiar con demencia en un centro geriátrico. A través de videollamadas se proporcionaba apoyo emocional, se enseñaba a los cuidadores a utilizar sus recursos $\mathrm{y}$, a desarrollar estrategias que les ayudasen a 
afrontar los problemas que surgiesen durante la transición a la institucionalización. Se midieron el nivel de culpabilidad, de carga, depresión, problemas con el personal y satisfacción con el centro. La intervención redujo el sentimiento de culpabilidad de los familiares en el grupo intervención y los problemas con el personal.

En este estudio se desconoce qué tipo de enmascaramiento se realizó. Además existe un riesgo elevado de que no tenga una potencia adecuada ya que, como reconocen los propios autores, el tamaño de la muestra puede ser insuficiente, lo cual impide extrapolar los resultados. Al igual que en otros estudios tuvieron un alto índice de abandono, pero se desconoce, ya que no se especifica, cómo lo manejaron para evitar sesgo en los resultados. Por tanto, se considera que este estudio presenta un riesgo alto de sesgo, pero sin embargo, la calidad del diseño, conduce a que se le otorgue un grado B de evidencia.

Por último, Tsai y $\mathrm{col}^{21} \mathrm{en}$ su estudio, a través de diseño cuasi-experimental, desarrollaron e implementaron una intervención consistente en videoconferencias entre los residentes y sus familiares. Su objetivo era disminuir los sentimientos de soledad y depresión de los residentes, aumentando la involucración de sus familiares en el centro. Hubiese sido interesante conocer si la intervención tenía efecto en los familiares, ya que solo analizaron los datos de los residentes. La intervención se implementó en un centro geriátrico en China, por lo que sería conveniente valorar las connotaciones culturales y sociales de dicho país. Por tanto, el diseño de este estudio impide analizar su calidad mediante la herramienta Cochrane. Sin embargo se puede señalar que obtuvo resultados significativos, aunque no se pueden extrapolar a otros contextos. La principal causa es la falta de aleatorización.

\section{DISCUSIÓN}

Esta revisión pone de manifiesto la escasez de investigaciones publicadas que han tratado de implementar y evaluar intervenciones familiares en centros geriátricos. Sin embargo, los estudios que se han llevado a cabo sugieren, que involucrar a la familia en las actividades de los centros geriátricos, es beneficioso para los residentes, la familia y para el personal sanitario ${ }^{9-11}$.

En general, podemos afirmar que la calidad metodológica de los estudios incluidos en la revisión es adecuada ya que el total de los estudios experimentales, a excepción del de Davies y col $^{20}$, han alcanzado un nivel $\mathrm{B}$ de evidencia. Incluso, aunque no se ha podido determinar la calidad de los estudios cuasi-experimentales de forma sistemática, se ha comprobado de manera crítica la adecuación en sus diseños.

Respecto a las intervenciones, la mayoría ${ }^{15-20}$ van dirigidas a trabajar con cuidadores familiares de ancianos institucionalizados, y de éstas, tres ${ }^{15-17}$ incluyen a su vez a los profesionales de los centros (enfermeras y auxiliares de enfermería).

En relación a las intervenciones enfocadas en los cuidadores informales ${ }^{18-21}$, como ya se ha comentado, su objetivo consistía en reducir las emociones negativas que pueden surgir durante el ingreso de su familiar en un centro. La literatura científica evidencia que la institucionalización en un centro geriátrico produce un gran impacto en la familia, siendo frecuente que el ingreso origine sentimientos de pérdida, tristeza, fracaso o culpabilidad ${ }^{10-11}$. En este sentido, las intervenciones analizadas en esta revisión han demostrado ser efectivas para mejorar estos sentimientos en los cuidadores. Este hecho resulta alentador e invita a seguir desarrollando intervenciones similares. Sin embargo sugerimos una serie de recomendaciones previas a futuras implementaciones. Por un lado, creemos conveniente poner en valor los diferentes perfiles de cuidadores familiares existentes. Es decir, cada individuo presenta una forma de reaccionar ante los problemas distinta $y$, un modo único de afrontamiento. Por ello debe considerarse si una intervención estandarizada puede ser adecuada para todos los participantes $22-23$. Por otro lado, y sumado a lo anterior, las intervenciones encontradas van dirigidas únicamente al cuidador principal o primario sin considerar al resto de miembros de la fami- 
lia, dando un enfoque individual, más que basado en la unidad familiar. Sin embargo, la evidencia científica demuestra que la enfermedad de un miembro afecta al resto de los miembros de la familia ${ }^{3-5,24}$. En este caso, y por el impacto que la institucionalización tiene sobre el sistema familiar, se recomienda llevar a cabo intervenciones con un enfoque sistémico familiar, de forma que la atención no se centre solamente en el cuidador principal, sino que vaya dirigida a la familia, considerando a ésta como unidad de cuidado.

En cuanto a las intervenciones dirigidas tanto a los cuidadores como a los profesionales de los centros ${ }^{15-17}$, su objetivo se centraba en mejorar la colaboración entre ambos, y con ello disminuir los conflictos entre ellos.

En este sentido las intervenciones han demostrado ser efectivas para las familias de los residentes. Sin embargo, el efecto que han causado en los profesionales ha sido moderado o nulo. A continuación se sugieren algunos aspectos que han podido ser la causa de estos resultados.

La evidencia muestra que es habitual que durante el ingreso en el centro la familia intente comunicarse con el personal y poner en su conocimiento los hábitos del residente así como los cuidados habituales que le proporcionaban en su domicilio ${ }^{11}$. Sin embargo, el personal de los centros no siempre está abierto a las sugerencias de la familia. Esta reacción puede desembocar en conflictos. El origen de estas tensiones puede ser explicado por una falta de formación de los profesionales de los centros, en la atención a la familia ${ }^{10-11}$.

Una de las causas de la falta de formación puede estar relacionada con la dificultad que entraña reclutar a los profesionales como participantes. La mayoría de ellos trabajan a turnos y poseen contratos temporales, por lo que les resulta complicado acudir a las sesiones de formación requeridas en los estudios ${ }^{15-17}$. Así mismo, la formación impartida a los profesionales podría haber sido insuficiente. La duración de los cursos ha sido, en total, de unas ocho horas ${ }^{15-17}$, identificándose una dificultad para impartir más sesiones, te- niendo en cuenta las circunstancias descritas anteriormente.

No obstante, debido a los beneficios que puede implicar este tipo de intervenciones, para ambos grupos, se anima a desarrollar futuras intervenciones que trabajen esta relación. Para ello se sugiere que, previo a su implementación, se busque el apoyo de la institución, de dichos centros, ya que su soporte facilitaría la participación de los profesionales ${ }^{25}$.

Otro aspecto que merece una especial consideración es el diseño de los estudios seleccionados. Los dos estudios cuasi-experimentales han mostrado que sus intervenciones son efectivas ${ }^{16,21}$. Sin embargo, los estudios que presentan un diseño experimental con grupo control han mostrado resultados más moderados. En este sentido, Zarit y coll $^{23}$ consideran que cuando se trata de evaluar intervenciones psicosociales, el diseño aleatorio experimental no siempre es el más adecuado. Esto es debido al rigor con el que se tratan las influencias externas en los resultados.

Así mismo, se aprecia cómo el perfil del profesional encargado de intervenir con los familiares suele ser el trabajador social o el psicólogo y no los profesionales de enfermería. Este hecho es paradójico, ya que el contacto directo con las enfermeras favorece las relaciones interpersonales, pudiendo establecer relaciones de colaboración y apoyo tanto con el residente como con su familia ${ }^{26,27}$.

Por último, se evidencia que los estudios seleccionados se han llevado a cabo en el contexto internacional, principalmente en los Estados Unidos. No obstante, es preciso puntualizar que esta revisión presenta una limitación en su metodología, debido a que se buscaron estudios publicados en bases de datos internacionales. Los autores reconocen que el presente trabajo podría presentar un sesgo de publicación, ya que los estudios seleccionados podrían no representar al total de estudios realizados. Por ejemplo, en España cada vez hay una tendencia mayor a reconocer la importancia que juega la familia en la institucionalización en centros geriátricos ${ }^{28}$. Por tanto, cabe la posibilidad de que se es- 
tén llevando a cabo intervenciones que no hayan sido publicadas en bases de datos internacionales.

En conclusión, podemos afirmar que esta revisión sistemática ha constatado la escasez de investigaciones publicadas sobre intervenciones familiares, desarrolladas en el ámbito de las residencias geriátricas. No obstante, la necesidad y el beneficio que supone involucrar a la familia en los centros geriátricos anima a seguir investigando en este campo. Para ello se considera necesario desarrollar estudios con diseños de elevada calidad metodológica. Asimismo, se recomienda que las intervenciones en los centros geriátricos tengan como foco del cuidado a la unidad familiar. Sin olvidar la importancia que, para llevar a cabo este tipo de cuidados, tiene el favorecer e impulsar la formación de los profesionales en la atención a la familia.

\section{BIBLIOGRAFÍA}

1. Ministerio de Trabajo y Asuntos Sociales. Libro Blanco: Atención a las Personas en Situación de Dependencia en España. Madrid. Ministerio de Trabajo y Asuntos Sociales, 2005.

2. IMSERSO. Las personas mayores en España. Datos Estadísticos Estatales y por Comunidades Autónomas (Vol. I). Madrid: Ministerio de Sanidad, Servicios Sociales e Igualdad 2012.

3. CANGa A. Experiencias de la familia cuidadora en la transición a la dependencia de un familiar anciano [Tesis doctoral]. Pamplona: Servicio de Publicaciones de la Universidad de Navarra; 2011.

4. CANGa A. Hacia una "familia cuidadora sostenible" An Sist Sanit Navar 2013; 36: 383-386.

5. CAnga A. Sobre los cuidadores de personas dependientes. An Sist Sanit Navar 2014; 37: 147-150.

6. IMSERSO. Las personas mayores en España. Datos estadísticos estatales y por comunidades autónomas (Vol. II). Madrid: Ministerio de Sanidad, Servicios Sociales e Igualdad 2012.

7. Dellasega C, Mastrian K. The process and consequences of institutionalizing an elder. West J Nurs Res 1995; 17: 123-136.

8. Sury L, BuRns K, Brodaty H. Moving in: Adjustment of people living with dementia going into a nursing home and their families. Int Psychogeriatr 2013, 25: 867-876.
9. Chang Y, Scheneider J. Decision-making process of nursing home placement among Chinese family caregivers. Persp in psych care 2010; 46: 108-118.

10. Davies S, Nolan M. 'Making the move': relatives' experiences of the transition to a care home. Health Soc Care Community 2004; 12: 517-526.

11. Davies S, Nolan M. 'Making it better': selfperceived roles of family caregivers of older people living in care homes: a qualitative study. Int J Nurs Stud 2006; 43: 281-291.

12. Harris J, Quatman C, Manring M, Siston R, FlantGAN D. How to write a systematic review. Clin Orthop Relat Res 2007; 455: 23-29.

13. HigGins JPT, GREen S (editores). Cochrane Handbook for Systematic Reviews of Interventions Version 5.1.0 The Cochrane Collaboration, 2011. Disponible en http:// www. cochrane-handbook.org [Consultado en agosto de 2014].

14. Nazir A, Unroe K, Tegeler M, Khan B, Azar J, Boustani M. Systematic review of interdisciplinary interventions in nursing homes. J Am Med Dir Assoc 2013; 14: 471-478.

15. Pillemer K, Suitor J, Henderson CR JR, Meador R, Schultz L, Robison J. A cooperative communication intervention for nursing home staff and family members of residents. Gerontologist 2003; 43: 96-106.

16. Maas M, Reed D, Park M, Specht J, Schutte D, KELLEY LS et al. Outcomes of family involvement in care intervention for caregivers of individuals with dementia. Nurs Res 2004; 53: 76-86.

17. Robison J, Curry L, Gruman C, Porter M, HenDERSON CR, PILLEMER K. Partners in caregiving in a special care environment: cooperative communication between staff and families on dementia units. Gerontologist 2007; 47: 504-515.

18. Ducharme F, Levesque L, Giroux F, Lachance L. Follow-up of an intervention program for caregivers of a relative with dementia living in a long-term care setting: Are there any persistent and delayed effects? Aging Ment Health 2005; 9: 461-469.

19. Gaugler J, Roth D, Haley W, Mittelman M. Modeling trajectories and transitions: results from the New York University caregiver intervention nurs res 2011; 60: 28-37.

20. Davis J, Tremont G, Bishop D, FortinsKy R. A telephone-delivered psychosocial intervention improves dementia caregiver adjustment following nursing home placement. Int J Geriatr Psychiatry 2011; 26: 380-387. 
21. Tsai H, Tsai Y, Wang H, Chang Y, Chu H. Videoconference program enhances social support, loneliness, and depressive status of elderly nursing home residents. Aging Ment Health 2010; 14: 947-954.

22. LOPEZ J, CRESPO M. Intervenciones con cuidadores de familiares mayores dependientes: una revisión. Psicothema 2007; 19: 72-80.

23. ZARIT S, Femia E. A future for family care and dementia intervention research? Challenges and strategies. Aging Ment Health 2008; 12 : 5-13.

24. Canga A, Vivar CG, Naval C. Dependencia y familia cuidadora: reflexiones para un abordaje familiar. An Sist Sanit Navar 2012; 34: 463469.

25. March S, Soler M, Miller F, Montaner I, PerezJaRAuTA MJ, Ramos M. Variabilidad en la implantación de las actividades comunitarias de promoción de la salud en España. An Sist Sanit Navar 2014; 37: 25-33.

26. BRAndBuRG G. Making the transition to nursing home life: a framework to help older adults adapt to the long-term care environment. J Gerontol Nurs 2007; 33: 50-56.

27. Gorlat-Sánchez B, García-Caro M, Peinado-Gorlat P, Expósito-Ruiz M, Quero-Rufián A, Cruz-QuintaNA F. Influencia del ámbito, rural o urbano, en la percepción de los apoyos y las necesidades de las personas cuidadoras en el hospital. An Sist Sanit Navar 2013; 36: 441-454.

28. Sociedad Española de Geriatría y Gerontología (SEGG). 100 Recomendaciones básicas de calidad y mejora continua para los servicios de centros de día de atención a personas mayores. 2004 Disponible en https:// www.segg.es/criterios_calidad.asp [Consultado en agosto de 2014]. 